\title{
La equidad y la imparcialidad en la reforma del sistema mexicano de salud
}

\author{
0 ctavio Gómez-Dantés, MC, MSP, ${ }^{(1)}$ Jesica Gómez-Jáuregui, Lic en CP, M en Econ, ${ }^{(2)}$ \\ Cristina Inclán, Lic en Soc. ${ }^{(3)}$
}

Gómez-Dantés O, Gómez-Jáuregui J, Inclán C.
La equidad y la imparcialidad en la reforma
del sistema mexicano de salud.
Salud Publica Mex 2004;46:399-416.

El texto completo en inglés de este artículo está disponible en: http://www.insp.mx/salud/index.html

\begin{abstract}
Resumen
Objetivo. Evaluar el impacto de la reforma de finales de la década de 1990 en la equidad e imparcialidad del sistema mexicano de salud. Material y métodos Para evaluar el impacto de esta reforma se utilizó un sistema de parámetros diseñado por Daniels y colaboradores que se adaptó a la realidad mexicana y al que se adicionaron indicadores específicos. Se hizo, finalmente, una revisión documental de la reforma para calificar su desempeño en cada uno de estos parámetros. Resultados. Excepto por las intervenciones en vivienda y nutricias, la reforma mostró poca preocupación por los determinantes de las condiciones de salud. En relación con la atención a la salud, las principales iniciativas de reforma tuvieron que ver con la ampliación de la cobertura de servicios esenciales y con la descentralización de los servicios de salud. Las actividades relacionadas con la justicia del financiamiento, la fragmentación del sistema, el énfasis en la atención curativa especializada, la rendición de cuentas y la transparencia fueron escasas. Conclusiones La reforma del sistema mexicano de salud de la segunda mitad de la década de 1990 tuvo cierto impacto sobre el acceso de los pobres a la atención a la salud y la eficiencia administrativa, pero un efecto mínimo sobre la justicia del financiamiento y el gobierno democrático. El texto completo en inglés de este artículo está disponible en: http:// www.insp.mx/salud/index.html
\end{abstract}

Palabras clave: reforma; equidad; imparcialidad; México

\author{
Gómez-Dantés 0, Gómez-Jáuregui J, Inclán C. \\ Equity and fairness \\ in the Mexican health system reform. \\ Salud Publica Mex 2004;46:399-416. \\ The English version of this paper \\ is available at: http://www.insp.mx/salud/index.html
}

\begin{abstract}
A bstract
Objective. To assess the equity and fairness of the Mexican health system reform that occurred in the late 1990's. Material and Methods The Mexican reform process was evaluated using the benchmark-system designed by Daniels et al. This benchmark system was adapted to the Mexican setting by adding specific indicators. A documentary review of the Mexican reform process was conducted to score its performance for each benchmark. Results Except for housing and nutrition components, the reform included few actions related to health determinants. For health care, the main reform initiatives were those related to extending the coverage of essential health services and decentralizing health care provision to the states. Reform initiatives included few activities related to fair financing, tiering, emphasis on second and third level care, accountability, and transparency. Conclusions.The late nineties reform of the Mexican health system had some positive effect on access of the poor to health care and administrative efficiency, but little impact on fair financing, quality of care, and democratic governance. The English version of this paper is available at: http://www.insp.mx/salud/index.html
\end{abstract}

Key words: reform; equity; fairness; Mexico

El desarrollo de esta investigación se financió con recursos del Consejo N acional de Ciencia y Tecnología de México.

(1) Dirección General de Evaluación del Desempeño, Secretaría de Salud, México, D F, México.

(2) Centro de Estudios Poblacionales y Salud Internacional, Universidad de Harvard Boston (MA), Estados Unidos de América.

(3) Centro de Investigaciones en Sistemas de Salud, Instituto N acional de Salud Pública, Cuernavaca, Morelos, México.

Fecha de recibido: 1 de agosto de 2003 - Fecha de aprobado: 23 de junio de 2004

Solicitud de sobretiros: Dr. 0 ctavio Gómez Dantés. Dirección General de Evaluación del Desempeño, Secretaría de Salud. Reforma 450, piso 12, colonia Juárez, México, DF, México.

Correo electrónico: ogomez@ salud.gob.mx 
$E$ n la década de los ochenta se implantaron en México diversas reformas estructurales que no pudieron reducir la incidencia de la pobreza ni modificar las desigualdades en la distribución del ingreso. A principios de esa década, 34\% de los hogares mexicanos eran pobres. En 1998 este porcentaje había ascendido a $38 \%{ }^{1, *}$ Las desigualdades en el ingreso tampoco se redujeron. A mediados de dicha década el 10\% de la población más rica del país disponía de $42.9 \%$ del ingreso nacional, mientras que el 10\% más pobre concentraba tan sólo $0.97 \% .^{2}$ En el año 2000, el 10\% más rico concentraba $42.4 \%$ del ingreso, mientras que el 10\% más pobre disponía apenas de 1.2\%.

La ampliación de la pobreza y la tenacidad de la desigualdad se reflejaron en las condiciones de salud: en la persistencia de daños pretransicionales (infecciones comunes, padecimientos relacionados con la mala nutrición y problemas asociados con la reproducción), sobre todo entre las poblaciones más pobres, y de brechas en las condiciones de salud y en el acceso a recursos y servicios para la salud inaceptables para un país de ingresos medios.

Con el fin de mitigar los efectos adversos más importantes de las crisis y de las medidas de ajuste, a principios de los años noventa se pusieron en marcha diversos programas e iniciativas dirigidos a atender las necesidades más apremiantes de las poblaciones vulnerables. En el sector salud estos programas e iniciativas buscaron mejorar la equidad, calidad y eficiencia de los servicios públicos, y en conjunto dieron lugar a lo que se dio en llamar "reforma del sector salud". ${ }^{3}$

Dentro de las diversas iniciativas de reforma destacan las siguientes:

\footnotetext{
* A estas cifras se llegó haciendo uso de la definición de pobreza de la Comisión Económica para América Latina y El Caribe (Cepal), que considera como pobre a quien percibe menos de 120 dólares por mes en las zonas urbanas y menos de 78 dólares en las rurales. Las cifras publicadas por la Secretaría de Desarrollo Social (Sedesol) para el año 2000 nos hablan de $24.2 \%$ de la población mexicana viviendo en condiciones de "pobreza alimentaria" (personas cuyo ingreso es insuficiente para cubrir sus necesidades de alimentación -entre 46 y 62 dólares mensuales en áreas rurales y urbanas, respectivamente-), $31.9 \%$ en condiciones de "pobreza de capacidades" (personas cuyo ingreso es insuficiente para cubrir sus necesidades de alimentación, educación y salud -entre 56 y 74 dólares mensuales en áreas rurales y urbanas, respectivamente-) y $45.9 \%$ en condiciones de "pobreza de patrimonio" (personas cuyo ingreso es insuficiente para cubrir sus necesidades de alimentación, salud, educación, vestido, calzado, vivienda y transporte público -entre 84 y 125 dólares mensuales en áreas rurales y urbanas, respectivamente-). Secretaría de Desarrollo Social. La medición de la pobreza en México. México, DF: Sedesol; 2002.
}

- La introducción, en 1997, de un nuevo esquema financiero dentro del Instituto Mexicano del Seguro Social (IMSS) que implicó el incremento de la participación gubernamental en su presupuesto de 4 a 39\%, con la consecuente reducción de la contribución obrero-patronal de 96 a 61\%.

- La desconcentración del IMSS mediante la creación de 139 áreas médicas que están recibiendo, desde enero de 1998, asignaciones presupuestales sobre la base de una fórmula capitada ajustada por edad y sexo. Estas áreas tienen la responsabilidad de ofrecer, con los recursos recibidos, servicios de primer y segundo nivel de atención a todos sus derechohabientes.

- La promoción de la afiliación al IMSS de los miembros de la economía informal con capacidad de pago a través de un nuevo plan financiado con contribuciones de los trabajadores y un subsidio federal.

- La conclusión del proceso de descentralización de los servicios de salud para la población no asegurada, iniciado formalmente en 1983, que implicó la devolución de la responsabilidad de la prestación de servicios a los estados.*

- La entrega de un paquete de 12 intervenciones a los mexicanos que hasta 1995 no contaban con acceso regular a servicios básicos de salud.

El propósito de este trabajo es evaluar el impacto de estas medidas de reforma en la equidad y justicia del sistema de salud. Para ello se utiliza una versión modificada de un grupo de parámetros que, a mediados de los años noventa, Daniels, Light y Caplan usaron para evaluar la preocupación por la equidad y la justicia de las propuestas de reforma que se generaron en los Estados Unidos de América (EUA) durante la primera administración del presidente Clinton. ${ }^{4}$

Es importante señalar que bajo el término "reforma del sistema mexicano de salud" se incluyen tanto las iniciativas antes descritas, que la administración del presidente Zedillo (1994-2000) agrupó bajo el término

\footnotetext{
* El 30 de agosto de 1983 se publica el decreto del Ejecutivo Federal que establece las bases a las que se sujetaría el Programa de Descentralización de los Servicios de Salud de la hasta entonces Secretaría de Salubridad y Asistencia. En esta primera fase, la descentralización se concretó en 14 estados. En 1996 se firma el Acuerdo Nacional para la Descentralización de los Servicios de Salud que permitió extender el proceso de descentralización a las 32 entidades federativas. A partir de 1998 los recursos descentralizados se transfieren a través del Fondo de Aportaciones para los Servicios de Salud (FASSA).
} 
de reforma, como diversas medidas aisladas que influyeron en el financiamiento, prestación y rectoría de los servicios de salud en México en la segunda mitad de los años noventa.

El objetivo del trabajo de investigación que dio origen a este artículo no fue generar información primaria sobre la desigualdad en las condiciones de salud y en la distribución de recursos y servicios para la salud en México, sino evaluar los esfuerzos de la reforma por abatir dichas desigualdades y construir un sistema de salud más justo. Para ello, se utilizó información secundaria generada tanto por las propias instituciones de salud como por diversas instituciones académicas y no gubernamentales.

Cabe destacar que los parámetros de Daniels y colaboradores forman parte de una serie de propuestas conceptuales, metodológicas y analíticas que, a finales de los años noventa y principios de esta década, diversos grupos académicos, varias agencias internacionales y algunas firmas consultoras generaron para evaluar el desempeño de los sistemas de salud. Dentro de estas iniciativas destacan, además del trabajo de Daniels y colaboradores, los trabajos de Knowles y colaboradores, Jee y Or, Murray y Frenk, y Hsiao y colaboradores. $^{5-8}$

\section{Material y métodos}

\section{Conceptos básicos}

De acuerdo con Whitehead y Dahlgren, ciertas diferencias -evitables, innecesarias e injustas-constituyen el meollo de las preocupaciones por la inequidad en salud. ${ }^{9}, 10$ Algunas desigualdades en salud son resultado de diferencias biológicas que no sabemos cómo evitar o atender. Otras son resultado de decisiones libres que tienen que ver con los estilos de vida. Hay otras más que tienen un carácter transitorio, como aquellas que son producto de comportamientos que promueven la salud y que son adoptados por un grupo antes que por otro (el cual estará en condiciones eventualmente de adoptar dichos comportamientos). Estos tres tipos de diferencias no plantean dilemas éticos. Hay otras diferencias, sin embargo, que constituyen casos paradigmáticos de inequidad injustificable. Se trata de aquellas diferencias que son producto de comportamientos nocivos para la salud en los que la capacidad de elección se ve severamente restringida: las que resultan de la exposición a condiciones de vida $y$ de trabajo insalubres y estresantes, y las que resultan de un acceso limitado a bienes y servicios esenciales de salud.
Así, la búsqueda de la equidad en salud supone el diseño e implantación de iniciativas que eviten la exposición a condiciones de vida y trabajo "no saludables", incrementen el acceso a los bienes y servicios esenciales de salud, y garanticen un financiamiento justo de la atención a la salud.

La equidad está íntimamente relacionada con la "imparcialidad". Según Rawls, la imparcialidad está vinculada al trato correcto entre personas que están cooperando o compitiendo. ${ }^{11}$ Se trata de personas libres, sin autoridad unas sobre otras, que reconocen las reglas que definen la competencia o la cooperación. Un sistema es imparcial cuando todos reconocen la forma y las condiciones bajo las cuales el sistema se pondrá en marcha y funcionará, y estas formas y condiciones son respetadas por todos.

Daniels y colaboradores incluyen todas estas consideraciones bajo el término imparcialidad (fairness, en inglés), que de acuerdo con ellos incluye la promoción de: a) la equidad (acceso de acuerdo con la necesidad y contribución de acuerdo con la capacidad de pago); b) la eficiencia (clínica y administrativa), y c) el gobierno democrático (información y transparencia, rendición de cuentas y empoderamiento de los ciudadanos).

En la evaluación de la reforma del sistema mexicano de salud, además de las consideraciones generales anteriores, se parte de reconocer que:

- En México existen desigualdades en salud que se ubican dentro de las diferencias que plantean dilemas éticos, resultado sobre todo de diferencias en las condiciones generales de vida, pero que también tienen que ver con diferencias en el acceso a los recursos y servicios para la salud.

- México es un país por lo menos formalmente preocupado por la justicia social en general y por la equidad en salud, como lo indican la Constitución de 1917 -que fue la primera Constitución que reconoció los derechos sociales- y, más específicamente, el Artículo $4^{\circ}$ constitucional, que garantiza el derecho a la protección de la salud.

- México es un país de ingresos medios que cuenta con los recursos suficientes para abatir en un periodo de tiempo relativamente breve sus iniquidades en salud.

- México es un país que cuenta con una madurez suficiente en sus instituciones como para conformar un sistema de salud imparcial.

\section{Metodología}

A principios de los años noventa, Daniels y colaboradores $^{12}$ desarrollaron una matriz con 10 parámetros y 
30 criterios para evaluar la preocupación por la equidad y la imparcialidad de diversas propuestas de reforma del sistema estadounidense de salud (cuadro I). Los parámetros y sus criterios permiten relacionar ciertas preguntas generales con medidas operativas para atenderlas. Varios de los parámetros cubren interrogantes relevantes para cualquier sistema de salud:

- ¿Qué barreras financieras existen en el acceso a servicios de salud?

- ¿Con qué servicios de atención a la salud se cuenta y qué tanto se han adecuado a las necesidades de la población?

- ¿Cómo están distribuidas las contribuciones para el pago de la atención a la salud?

- ¿Cómo promueve el sistema la eficiencia clínica y administrativa de manera que los presupuestos de salud produzcan valor por el dinero?

- ¿Qué tan responsables ante el público son las instituciones de atención a la salud?

- ¿Qué tipos de libertad de elección pueden ejercer los usuarios dentro del sistema?

No obstante, algunos parámetros dedican demasiada atención a aspectos muy específicos del sistema de salud estadounidense. Así también, en este sistema de parámetros están ausentes criterios para evaluar la contribución a la imparcialidad y la equidad de propuestas de reforma de países en desarrollo.

Fue por estas dos razones que en este proyecto se modificaron los parámetros originales para aplicarlos al análisis de la reforma del sector salud mexicano. En el proceso de diseño del sistema de parámetros para México se siguieron los siguientes pasos:

1. Se adaptó el sistema de parámetros original al contexto mexicano, eliminando o limitando la importancia de aquello que no resultaba particularmente relevante e incorporando criterios para evaluar medidas que posiblemente no hubiesen sido dignas de atención en el proceso de reforma de EUA. Esto dio como resultado ocho parámetros y 27 criterios (cuadro II).

2. En consulta con expertos, se definieron indicadores de referencia para los parámetros. La evolución de sus valores se utilizaron como medidas aproximadas de impacto de las acciones de reforma. Cabe señalar, no obstante, que estos valores deben tomarse con cautela, dados los problemas de la calidad de la información y su dependencia de otras acciones extrasectoriales.

3. A partir de la revisión exhaustiva del sistema mexicano de salud y su reforma, y de la revisión de los

\section{Cuadro I \\ Parámetros para la evaluación DE LA IMPARCIALIDAD Y LA EQUIDAD \\ EN LAS PROPUESTAS DE REFORMA DEL SISTEMA DE SALUD de Estados Unidos de AmÉrica}

Parámetro I. Universalidad en el acceso y cobertura

- Cobertura obligatoria

- Posibilidad de transferir la cobertura sin perder la continuidad

Parámetro II. Universalidad en el acceso, minimización de barreras no financieras

- Minimización de la mala distribución de personal, instalaciones e insumos

- Reforma a la educación del profesional de la salud

- Minimización de las barreras lingüísticas, culturales y de clase

- Minimización de barreras educativas y de información

Parámetro III. Beneficios integrales y uniformes

- Integralidad: todos los servicios necesarios y efectivos que se consideran financiables

- No exclusión categórica de servicios tales como salud mental y atención en asilos

- Reducción del escalonamiento y calidad uniforme

- Beneficios no dependientes de ahorros

Parámetro IV. Financiamiento equitativo - contribución con base en grupos

- Determinación de pólizas con base en grupos

- Discriminación mínima vía pagos en efectivo

Parámetro V. Financiamiento según capacidad de pago

- Todos los pagos directos e indirectos y los pagos por servicio ajustados al presupuesto del hogar y capacidad de pago

Parámetro VI.Valor por el dinero - eficacia clínica

- Enfasis en la atención de primer nivel

- Enfasis en salud pública y prevención

- Evaluación sistemática de los resultados

- Minimización de la sobre y subutilización

Parámetro VII.Valor por el dinero - eficiencia financiera

- Minimización de gastos de administración

- Estricta negociación de contratos

- Minimización de subsidios de costos entre dependencias

- Medidas antifraude y antiabuso

Parámetro VIII. Responsabilidad ante el público

- Procedimientos explícitos, públicos y detallados para la evaluación de los servicios, con informes públicos

- Procedimientos explícitos y democráticos para la asignación de recursos

- Procedimientos justos para quejas y reclamos

- Protección adecuada de la privacía

Parámetro IX. Comparabilidad

- Presupuestos para la salud diseñados de modo que puedan ser comparados

Parámetro X.G rado de libertad de elección de los usuarios

- Elección del proveedor de servicios de primer nivel

- Elección de especialistas

- Elección de otros proveedores de salud

- Elección de los procedimientos

salud pública de méxico / vol.46, no.5, septiembre-octubre de 2004 


\section{Cuadro II \\ Parámetros para eValuar la preocupación POR LA EQUIDAD Y EVALUACIÓN EN LA REFORMA MEXICANA DEL SISTEMA DE SALUD}

Parámetro I. Salud pública intersectorial

- Nutrición básica

- Vivienda

- Factores ambientales

- Violencia y seguridad pública

- Educación y educación para la salud

- Desarrollo de información para monitorear la inequidad en salud

Parámetro II. Universalidad en el acceso, minimización de barreras financieras

- Cobertura del sector informal

- Aseguramiento del sector formal

Parámetro III. Universalidad en el acceso, minimización de barreras no financieras

- Minimización de la mala distribución de recursos

- Minimización de las barreras de género

- Minimización de las barreras de lengua, culturales y de clase

Parámetro IV. Beneficios integrales y uniformes

- Integralidad: acceso a todos los servicios necesarios y efectivos que se consideran financiables

- Reducción del escalonamiento y calidad uniforme

Parámetro V. Financiamiento equitativo

- Justicia en la contribución financiera

Parámetro VI. Eficiencia distributiva, efectividad y calidad de la atención

- Enfasis en la atención de primer nivel

- Prácticas basadas en evidencia

- Certificación de los profesionales de la salud

- Medidas de acreditación de planes y hospitales

- Participación comunitaria en la búsqueda de la calidad de la atención

Parámetro VII.Valor por el dinero - eficiencia financiera

- Minimización de gastos de administración

- Compras que reduzcan costos

- Minimización del desplazamiento de costos

- Medidas antifraude y antiabuso

Parámetro VIII. Responsabilidad ante el público

- Procedimientos explícitos, públicos y detallados para la evaluación de los servicios, con informes públicos

- Procedimientos explícitos y democráticos para la asignación de recursos

- Procedimientos justos para quejas y reclamos

valores recientes de los indicadores correspondientes, se asignaron calificaciones cualitativas a los diversos criterios de cada parámetro $(+,++\mathrm{y}+++)$ que ayudaron a evaluar el desempeño del sistema en relación con la equidad y la imparcialidad.

Los resultados de este ejercicio también se utilizaron como insumos en un artículo que discute la utilización de estos parámetros en países en desarrollo. ${ }^{12}$

\section{Resultados}

\section{Parámetro I. Salud pública intersectorial}

En este parámetro se evaluaron las acciones emprendidas en el terreno de la nutrición, vivienda, factores ambientales, violencia y seguridad pública, educación y la generación de información para monitorear la iniquidad en salud. Aunque el sector salud no es directamente responsable de lo que sucede en estos terrenos, sus obligaciones como instancia rectora incluyen el cabildeo alrededor de actividades extrasectoriales que tienen un impacto sobre la salud.

\section{Nutrición básica}

En el terreno de la nutrición destaca la puesta en marcha del Programa de Educación, Salud y Alimentación (Progresa), que entre 1997 y 2000 ofreció apoyos financieros directos a cambio de la adhesión a intervenciones nutricias, de salud y educativas. Los beneficios de este programa se diseñaron de tal manera que no sólo incrementaron los recursos financieros del hogar sino que promovieron la participación de las familias en actividades que ampliaron su capital humano.

Progresa proporcionó suplementos nutricios a todos los niños de entre 4 y 24 meses de edad de la población blanco, a los niños de 3 a 5 años de edad desnutridos, y a las mujeres de bajo peso, embarazadas y lactando. A finales de 2000, este programa, que contó con un presupuesto de 800 millones de dólares (0.2\% del PIB), estaba beneficiando a 2.6 millones de familias de 50 mil comunidades, que representan 30\% de los hogares rurales y $10 \%$ de todas las familias de México. ${ }^{13}$ Los resultados de una evaluación externa mostraron un descenso de $18 \%$ en la prevalencia de anemia y un incremento de 1 a $4 \%$ en la talla de los niños menores de cinco años de edad de la población blanco. ${ }^{13}$

El impacto a escala nacional de este y otros programas (Programa de Desayunos Escolares, Programa de Asistencia Social Alimentaria a Familias, Programa de Cocinas Populares y Unidades de Servicios Integrales, Leche Industrializada y Programa de Subsidio al Consumo de Tortilla) ha sido muy variable. La última Encuesta Nacional de Nutrición arroja cifras muy desiguales en los principales indicadores nutricios (cuadro III). ${ }^{14}$ En la última década disminuyó la prevalencia de bajo peso para la edad en menores de cinco años de edad, que sin embargo sigue siendo elevada $(17.8 \%)$. La prevalencia de anemia en mujeres en edad 


\section{Cuadro III \\ VALORES DE LOS INDICADORES DE LOS PARÁMETROS PARA EL SEGUIMIENTO DE LA EQUIDAD Y LA IMPARCIALIDAD EN LA REFORMA DEL SISTEMA MEXICANO DE SALUD}

Parámetro/Indicador

1990

1999

Parámetro I. Salud pública intersectorial

Nutrición básica

- Prevalencia de anemia en mujeres en edad reproductiva*

- Prevalencia de bajo peso por edad en $<5$ años*

$\quad 20.2 \%$

- Prevalencia de obesidad en mujeres de 12 a 49 años de edad*

$22.8 \% \quad 17.8 \%$

Vivienda

- \% de la población que vive en condiciones de hacinamiento

- \% de hogares con piso de tierra

$51 \% \quad 45.9 \%$

$19.6 \% \quad 13.4 \%$

Educación y educación para la salud

- Analfabetismo en población mayor de 15 años de edad

$12.4 \% \quad 9.5 \%$

- Escolaridad promedio en $>15$ años de edad

- Prevalencia de tabaquismo en personas de 12 a 17 años de edad $\$$

$\begin{array}{ll}6.4 \text { años } & 7.5 \text { años } \\ \text { ND } & 8.7 \%\end{array}$

Seguridad vial y pública

- Tasa de mortalidad por accidentes de tráfico (por 100000 habitantes)\#

$17.2 \quad 11.9$

- Tasa de mortalidad por homicidios (por 100000 habitantes)\#

17.8

12.5

Factores ambientales

- $\quad \%$ de la población con acceso a agua potable

- \% de la población con acceso a infraestructura sanitaria

- Indice de calidad del aire (promedio del DF, Guadalajara y Monterrey)\&

\begin{tabular}{cl}
$78 \%$ & $83.8 \%$ \\
\hline $61 \%$ & $78.5 \%$ \\
\hline ND & 17 (malo)
\end{tabular}

Parámetro III. U niversalidad en el acceso/barreras no financieras

Distribución de recursos físicos y humanos

- Camas por 10000 habitantes $^{*}$

- Médicos por 1000 habitantes $^{\neq}$

$11.4 \quad 7.9$

Género

- Indice de desarrollo de género\&

1.36 1.17

- Mortalidad materna (por 10000 nacidos vivos registrados) ${ }^{6}$ 0.778

Parámetro V. Financiamiento equitativo

Justicia en el financiamiento de los servicios de salud

- Indice de justicia financiera

ND 0.903

- \% de hogares con gastos catastróficos por motivos de salud

ND
$11 \%$

* Referencia 14. Las cifras que por razones de presentación aparecen como de 1990, corresponden en este caso a 1988

‡ Referencia 15

$\S$ Referencia 16

\# Referencia 17

\& Referencia 18. El índice de calidad del aire agrega valores de seis indicadores: bióxido de azufre, bióxido de nitrógeno, ozono, monóxido de carbono, partículas que dañan a la salud y reducen la visibilidad, y plomo

* Referencia 19

- Referencia 20

- Referencia 21

\$ Referencia 22 
reproductiva (12 a 49 años) aumentó de 15.6\%, en 1988, a $20.2 \%$, en 1999 . Un hallazgo alarmante de esta encuesta es el incremento de la prevalencia de sobrepeso y obesidad, que entre las mujeres en edad reproductiva supera ya $50 \%$.

\section{Vivienda}

En el rubro de vivienda, las principales acciones desarrolladas en años recientes incluyen el otorgamiento de 2 millones de créditos para la adquisición y mejoramiento de la vivienda, la construcción de 11000 viviendas en localidades marginadas de los estados de Veracruz, Chihuahua, Hidalgo y México, y la implantación de programas para la ampliación del suelo de vivienda. Conviene destacar que en la última década el porcentaje de hogares que vive en condiciones de hacinamiento disminuyó de 51 a $45.9 \%$ y que el porcentaje de hogares con piso de tierra descendió de 19.6 a $13.4 \% .^{15,23}$

\section{Factores ambientales}

Por lo que se refiere al agua potable y al saneamiento básico, sus coberturas aumentaron de 78 a $83.8 \%$ en el primer caso, y de 61 a $73 \%$ en el segundo entre 1990 y $2000 .{ }^{15,24}$

En el rubro ambiental destacan las siguientes acciones: a) el diseño de la Norma Oficial para la Calidad del Aire, que incluye criterios para la evaluación de la calidad del aire de acuerdo con el tipo y cantidad de partículas suspendidas; b) la puesta en marcha del Programa para Mejorar la Calidad del Aire en seis grandes ciudades del país, y c) la implantación del Programa de Regulación Ambiental de Higiene y Trabajo, acoplado al Plan de Contingencia Ambiental. ${ }^{25}$ En el caso de la Ciudad de México, estas medidas redujeron el porcentaje de días con niveles de contaminación inaceptables de 96\% en 1991 a 89\% en 1996. El número de días de contingencia ambiental también se redujo de 56, en 1991, a siete, en 1995, y a cero en el año $2000 .^{26}$

\section{Violencia y seguridad pública}

En el rubro de seguridad vial y pública destacan la reactivación del Consejo para la Prevención de Accidentes, en el que participan las Secretarías de Salud y de Comunicaciones y Transporte, y el diseño de la Norma Oficial Mexicana para la Violencia, que intenta facilitar la identificación de lesiones debidas a actos violentos. Localmente, el gobierno del Distrito Federal creó un Sistema para la Atención y Prevención de la Violencia Doméstica en la Ciudad de México; puso en marcha el Programa de Seguridad Ciudadana que implica el aumento de policías en zonas específicas de la ciudad con apoyo de "Comités Ciudadanos" que vigilan sus actividades, y creó la Policía Federal Preventiva como organismo especializado en el combate a la delincuencia y el crimen organizado. ${ }^{27}$

Los indicadores de accidentes y violencia muestran mejoras que deben tomarse con cautela, dados los problemas de registro. La tasa de mortalidad por accidentes -la gran mayoría accidentes de tráfico- se redujo de 48.5 por 100000 habitantes en 1990 a 35.8 en $2000 .^{28} \mathrm{La}$ tasa de mortalidad por homicidios disminuyó de 17.8 por 100000 habitantes en 1990 a 10.6 en $2000 .^{28}$

\section{Educación y educación para la salud}

En el tema de actividades de promoción de la salud, tan importantes ahora que las principales causas de daños a la salud están estrechamente ligadas a los estilos de vida, se hará mención a las acciones emprendidas en materia de VIH-SIDA, tabaco y drogas.

En el caso del VIH-SIDA destacan las siguientes acciones implantadas por la Secretaría de Educación Pública y el CONASIDA: a) la distribución en escuelas de educación superior de información relativa al uso del condón y la prueba de VIH-SIDA; b) la introducción en secundaria de la asignatura "orientación educativa", con temas relativos a la salud, la sexualidad y las infecciones de transmisión sexual, y c) la publicación de libros para padres de familia sobre la sexualidad de los hijos y el consumo de sustancias tóxicas. ${ }^{29,30}$

En consumo de tabaco, el Consejo Nacional contra las Adicciones (Conadic) echó a andar el Programa contra el Tabaquismo. Entre las instituciones participantes se encuentran los programas estatales de las Secretarías de Salud y de Educación Pública, diversas organizaciones no gubernamentales y varios organismos privados.

En materia de consumo de alcohol y drogas, Conadic estableció en 1997 el Programa de Prevención y Control de Adicciones. Entre sus acciones destacan la inserción de contenidos preventivos en 13 millones de libros de texto gratuitos y la transmisión de campañas de prevención en radiodifusoras y televisión. ${ }^{31}$

Los indicadores relacionados con estos rubros no son alentadores. La prevalencia del consumo de tabaco entre la población de 12 y 65 años de edad aumentó de $25.8 \%$ en 1988 a $27.7 \%$ en $1998 .{ }^{16}$ El consumo de droga -alguna vez- en el grupo de edad de 12 a 65 años también aumentó de 3.9\%, en 1993, a 5.3\% en 1998. El incremento en el consumo de cocaína es particularmente alarmante. 
Desarrollo de información para monitorear

la inequidad en salud

Por último, en lo que se refiere al monitoreo de la iniquidad en salud, las actividades desarrolladas por las autoridades de salud fueron prácticamente nulas. Los centros académicos -El Colegio de México, el Instituto Tecnológico Autónomo de México- y algunos centros de análisis no gubernamentales -la Fundación Mexicana para la Salud- fueron los que estuvieron documentando en la segunda mitad de la década de 1990 las desigualdades en salud y sus determinantes.

\section{Parámetro II. Universalidad en el acceso: minimización de barreras financieras}

Cualquier sistema de salud preocupado por la equidad debe tratar de garantizar un acceso universal a ser- vicios básicos de salud. En el caso mexicano este acceso lo tienen garantizado la clase media y los afiliados a las instituciones de seguridad social, como el Instituto Mexicano del Seguro Social (IMSS), Instituto de Seguridad y Servicios Sociales de los Trabajadores del Estado (ISSSTE) y Petróleos Mexicanos (Pemex), es decir, los trabajadores de la economía formal, que represen$\tan$ aproximadamente $40 \%$ de la población (figura 1). La población del sector informal -alrededor de $50 \mathrm{mi}-$ llones de personas que hacen uso sobre todo de los servicios ofrecidos por la Secretaría de Salud- tiene un acceso limitado.

\section{Cobertura del sector informal}

De acuerdo con cifras oficiales, en 1995, 10\% de la población no asegurada de México (10 millones de personas) carecía de acceso regular a servicios básicos de

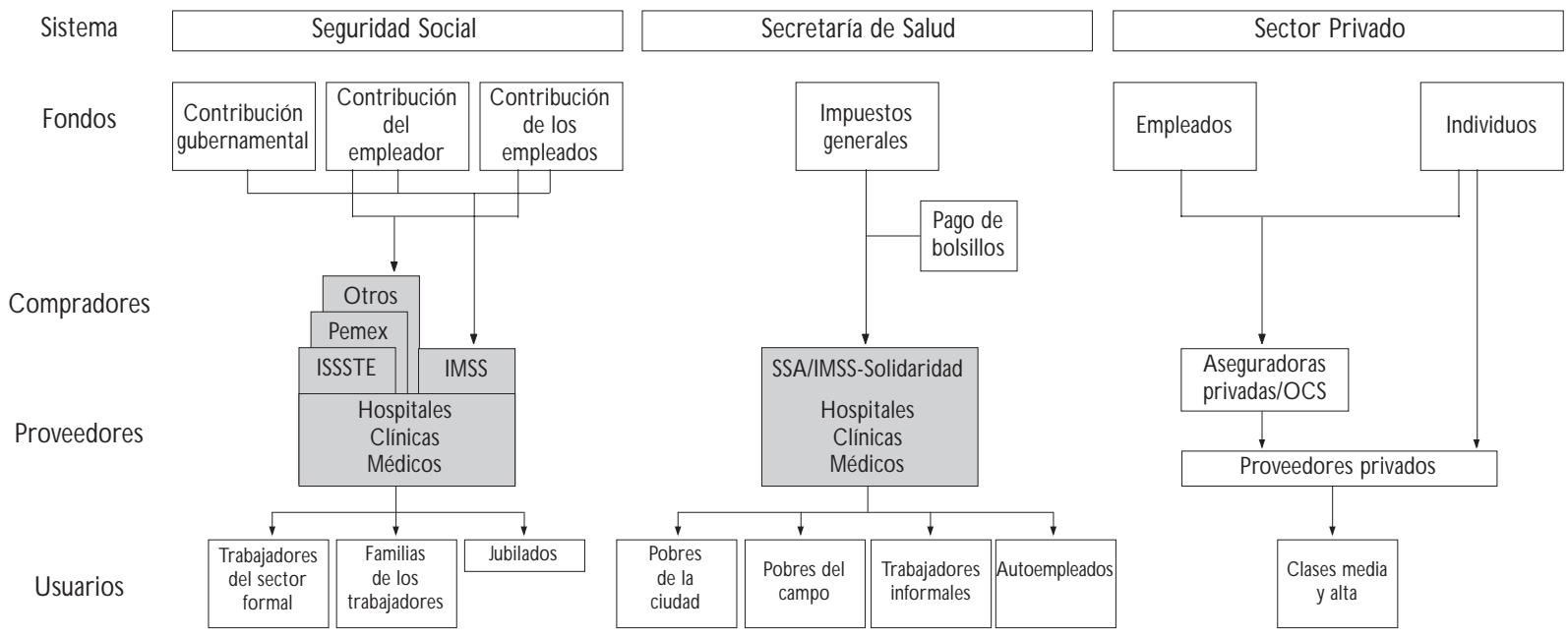

Pemex: Petróleos Mexicanos

ISSSTE: Instituto de Seguridad y Servicios Sociales de los Trabajadores del Estado

IMSS: Instituto Mexicano del Seguro Social

SSA: Secretaría de Salud

OCS: O rganizaciones de Conservación de la Salud

\section{Figura 1. Los tres componentes del sistema mexicano de salud: Seguridad social, Secretaría de Salud y SECTOR PRIVADO.*}

\footnotetext{
* El primer componente incluye a las instituciones que prestan servicios a la población no asegurada -alrededor de 50\% de la población total-, en su mayoría pobres del campo y de la ciudad. Las agencias más importantes de este componente son la Secretaría de Salud y el Programa IMSS-Solidaridad. El segundo componente es el sistema de seguridad social, que se encarga de prestar atención a poco más de $40 \%$ de la población. Este componente incluye al IMSS, que tiene a su cargo a los trabajadores del sector formal de la economía, y diversas organizaciones para los empleados públicos (ISSSTE), las fuerzas armadas y los trabajadores de la compañía de petróleos. El tercer componente es el sector privado, que está formado por una gran diversidad de prestadores que trabajan en consultorios, clínicas ambulatorias, hospitales y unidades de medicina tradicional, en su mayoría sobre una base lucrativa. Cabe señalar que tanto la población asegurada como no asegurada hacen uso regular de los servicios de salud privados. La población asegurada también utiliza con regularidad los servicios de las instituciones para población no asegurada.
} 
salud. Con el fin de enfrentar este problema, la Secretaría de Salud implantó el Programa de Ampliación de Cobertura (PAC), que ofreció entre 1996 y 2000 un paquete de 12 intervenciones a esos 10 millones de mexicanos. ${ }^{* 32}$ La oferta de servicios para ciertas poblaciones pobres que ya contaban con cierto nivel de atención también se reforzó a través de este programa. A mediados del año 2000, según los responsables del programa, el PAC había alcanzado a prácticamente toda su población blanco, lo que significaba que de cada 100 mexicanos, 99 tenían acceso por los menos a servicios esenciales de salud. Esta afirmación resulta, sin embargo, difícil de sustentar, ya que nunca se pusieron a disposición del público los resultados de algún esfuerzo de evaluación que demostrara este nivel de logro. De hecho, se sabía que la cobertura de las intervenciones del paquete era muy irregular y que un número importante de personas recibía servicios básicos de salud de manera muy esporádica, a través de brigadas móviles. ${ }^{33}$

El PAC en las comunidades Progresa se constituyó en el componente de salud de este último programa. Esta combinación tenía la ventaja de que las familias afiliadas a Progresa, para recibir el subsidio monetario, tenían que demostrar, entre otras cosas, comprobantes de atención prenatal, vigilancia del crecimiento y desarrollo, inmunizaciones, consultas preventivas periódicas para adultos y educación para la salud.

Además del PAC, con el fin de ampliar la cobertura del sistema mexicano de salud, en la segunda mitad de los años noventa se implantó también el Seguro de Salud para la Familia. Gracias a este seguro, una familia del sector informal de la economía puede acceder a los servicios del IMSS a través de una cuota familiar, que varía según el nivel de ingresos entre 100 y 250 dólares anuales, al que se asocia un subsidio gubernamental de aproximadamente 110 dólares. Hacia finales de 2000, el número de beneficiados por este seguro ascendía a 2.5 millones. ${ }^{34}$ Las posibilidades de ampliar más este seguro, sin embargo, son muy pocas, ya que sólo un porcentaje muy pequeño de la población del

\footnotetext{
* El paquete incluía las siguientes intervenciones: a) saneamiento básico familiar, b) planificación familiar, c) atención prenatal, del parto y del puerperio, y del recién nacido, d) vigilancia de la nutrición y crecimiento infantil, e) inmunizaciones, f) manejo de casos de diarrea en el hogar, g) tratamiento anti-parasitario a las familias, h) manejo de infecciones respiratorias agudas, i) prevención y control de la tuberculosis pulmonar, j) prevención y control de la hipertensión arterial y de la diabetes mellitus, k) prevención de accidentes y manejo inicial de lesiones, y l) capacitación comunitaria para el autocuidado de la salud. A este paquete se sumó poco despues la detección y el tratamiento del cáncer cervicouterino.
}

sector informal estaría en condiciones de pagar esta cuota, como lo muestran las cifras de pobreza que se presentan al inicio de este trabajo.

\section{Parámetro III. Universalidad en el acceso: Minimización de barreras no financieras}

La eliminación de las barreras financieras a los servicios de salud no garantiza necesariamente su utilización, ya que pueden existir barreras geográficas, organizacionales, culturales, de clase y de género.

\section{Minimización de la mala distribución de recursos}

A los obstáculos de tipo financiero de los sistemas de salud habría que agregar, en primer lugar, los problemas de escasez y mala distribución de recursos. En México, en 1995 había tasas de 0.5 consultorios, 0.8 camas y 0.06 salas de expulsión por 1000 habitantes, que son cifras muy modestas. ${ }^{35}$ Pero esta situación se complicaba todavía más por los problemas de distribución. Sonora y Baja California contaban con 1.0 y 1.5 camas por 1000 habitantes, respectivamente, mientras que Puebla y Tlaxcala contaban con sólo 0.5. ${ }^{34}$ En Aguascalientes, Baja California Sur, Coahuila, Colima, Distrito Federal y Sonora el porcentaje de nacimientos en instituciones del sector ascendía a más de $90 \%$, mientras que en Chiapas, Guerrero, Estado de México, Oaxaca y Puebla era menor de $50 \% .^{35}$

El Programa de Apoyo a los Servicios de Salud para Población Abierta (PASSPA 1991-1995) y el PAC trataron de atender estos problemas. El primero de estos programas construyó una gran cantidad de obra y amplió la oferta de recursos humanos para la salud, lo que permitió incrementar la cobertura potencial de los servicios de salud para población no asegurada en Chiapas, Guerrero, Hidalgo y Oaxaca en cerca de 60\% entre 1990 y $1994 .{ }^{36}$

\section{Minimización de las barreras de género}

Es muy poca la información con la que se cuenta para documentar las barreras de género que existen en el sistema mexicano de salud. Sin embargo, de manera indirecta se puede tener una idea de los problemas específicos de acceso a servicios de salud que enfrentan las mujeres. Uno de ellos es el acceso a la atención del parto por personal calificado, problema del que ya se habló. Esto ha dado lugar a cifras de mortalidad materna inaceptables para un país de ingresos medios altos: 8.4 muertes maternas -la mayoría por causas obstétricas directas- por 10000 nacidos vivos en $19900^{37}$ En 1999 esta tasa ascendía a poco más de $8 .^{38}$ 
El cáncer cérvico-uterino $(\mathrm{CaCu})$, por su parte, producía en 1995 poco más de 21 muertes por 100000 mujeres de 25 años de edad y más, que era una de las cifras más altas del mundo. ${ }^{37}$ Esto a pesar de que México cuenta, desde 1974, con un Programa de Detección Oportuna de Cáncer Cérvico Uterino. Este programa, sin embargo, sufre de una baja utilización de servicios debido, entre otras cosas, a barreras culturales y geográficas, mal trato a las usuarias, mala calidad de las muestras y de su interpretación, y baja notificación de resultados ${ }^{39}$ A pesar de esto la tasa de mortalidad por $\mathrm{CaCu}$ descendió ligeramente a 19.7 en 1999.38

Entre las acciones con enfoque de género que se implantaron durante los años de reforma destaca el Programa de Salud Reproductiva y Planificación Familiar 1995-2000, que incluyó acciones en materia de educación sexual, planificación familiar, cuidado a la salud de las mujeres, atención materno-infantil y salud preventiva. Gracias a este Programa se incrementó el uso de anticonceptivos y las actividades de auto cuidado de la salud. Sin embargo, el control de la natalidad todavía refleja diferencias de género, como lo demuestra el hecho de que la gran mayoría de los métodos anticonceptivos promovidos no requieren de la participación del hombre.

\section{Minimización de las barreras de lengua, culturales $y$ de clase}

Los hablantes de lengua indígena de México de cinco años de edad y más representan $7.3 \%$ de la población total (6.3 millones de hablantes de lengua indígena). ${ }^{40}$ Están concentrados sobre todo en los estados de Chiapas, Hidalgo, Oaxaca, Veracruz y Yucatán, en su mayoría en municipios de alta y muy alta marginalidad. Esto se refleja en sus condiciones de salud. La tasa de mortalidad materna en los municipios indígenas de Chiapas, Guerrero, Hidalgo y Oaxaca es de 11, 28.3, 9.1 y 12 por 10000 nacidos vivos, respectivamente; en contraste, las cifras estatales para estas mismas entidades son de 7, 7, 5.4 y 5.8 , respectivamente. ${ }^{22}$ La prevalencia de desnutrición crónica en el ámbito nacional es de $17.7 \%$, mientras que en la población indígena es de $44 \%{ }^{14} \mathrm{Las}$ mismas diferencias se observan con los recursos. En Chiapas, en 1995, 42\% de los partos se atendían en unidades de salud, en contraste con menos de $35 \%$ en las zonas indígenas de esta misma entidad. ${ }^{41}$

Algunas de estas carencias se enfrentaron a través de Progresa, PASSPA y PAC. Estos dos últimos programas ampliaron la infraestructura y los recursos humanos para la salud, y, por lo mismo, la cobertura de servicios básicos en las zonas indígenas, incluyendo la atención del parto. ${ }^{36}$
Por lo que se refiere a la discriminación sexual, está siendo lenta pero consistentemente superada gracias al alto nivel de organización de los grupos relacionados con el VIH-SIDA. No obstante, $25 \%$ de las quejas que los usuarios de los servicios de salud presentan ante las Comisiones Nacional y Estatales de Derechos Humanos se relacionan con negligencia médica y negación del servicio, y los enfermos de VIH-SIDA son los principales afectados. ${ }^{42}$

\section{Parámetro IV. Beneficios integrales y uniformes}

Integralidad: acceso a todos los servicios necesarios y efectivos que se consideran financiables

La exclusión de poblaciones es producto de la fragmentación del sistema de salud. El acceso a la seguridad social ha estado históricamente limitado a los miembros de la economía formal. Los autoempleados y los trabajadores rurales han sido menos beneficiados y han recibido un paquete de servicios considerablemente menor y de menor calidad. El caso más extremo son los 10 millones de personas que en 1995 no contaban con acceso regular a servicios básicos de salud.

En 1995, para enfrentar específicamente este problema, se implantó el PAC, que, como ya mencionamos, ofreció un paquete de 12 intervenciones básicas a una población objetivo que estaba concentrada en municipios de muy alta marginación de 19 estados de la república. El IMSS, por su parte, implantó el Seguro de Salud para la Familia, dirigido a los miembros de la economía informal con capacidad de pago, que también se discutió con anterioridad.

\section{Reducción del escalonamiento y calidad uniforme}

A pesar de los esfuerzos de ampliación de cobertura, la reforma no sólo no ha podido reducir la fragmentación del sistema, sino que incluso la ha aumentado, como se muestra en el cuadro IV, en donde se identifican las diversas poblaciones, por un lado, y las instituciones y programas de atención, por otro.

Esta fragmentación se asocia además a un gasto y un paquete de beneficios muy diferentes. La diferencia en el gasto per cápita entre los servicios médicos de Pemex y otras instituciones de salud es de 2 a 6 veces. ${ }^{22}$ La relación entre el gasto per cápita del IMSS y el gasto per cápita de la Secretaría de Salud en el año 2000 es de casi 2 a 1. Además habría que considerar que el gasto de la Secretaría de Salud incluye gastos en actividades de rectoría y vigilancia epidemiológica que benefician a toda la población, mientras que el gasto de 


\section{Cuadro IV \\ Segmentos del sistema mexicano de SAlud}

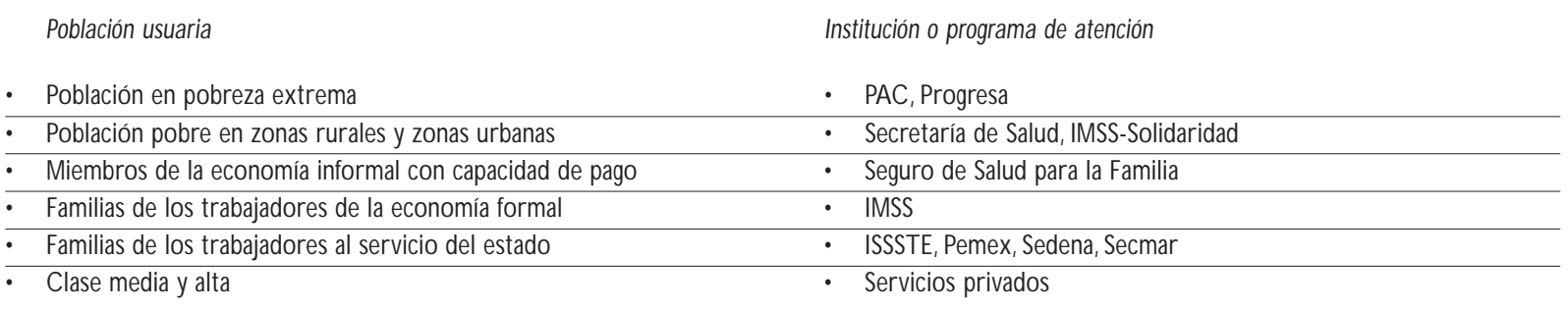

PAC: Programa de Ampliación de Cobertura

IMSS: Instituto Mexicano del Seguro Social

ISSSTE: Instituto de Seguridad y Servicios Sociales de los Trabajadores del Estado

Pemex: Petróleos Mexicanos

Sedena: Secretaría de la D efensa N acional

Secmar : Secretaría de Marina

las instituciones de seguridad social, que es sólo gasto en atención médica, beneficia exclusivamente a sus derechohabientes.

\section{Calidad uniforme}

La segmentación también trae consigo diferencias importantes en la calidad de la atención. En un estudio realizado en la década de los noventa, se demostró que un niño nacido en un hospital de la Secretaría de Salud corría un riesgo tres veces mayor de fallecer en los primeros siete días de vida que un niño del mismo peso nacido en un hospital del IMSS. ${ }^{43}$ Los investigadores concluyeron que estas diferencias se debían fundamentalmente a diferencias en la calidad de la atención. Poco, sin embargo, se hizo específicamente en este rubro entre 1995 y 2000 en México, salvo documentar el problema de la mala calidad de los servicios a través del Diagnóstico Basal de Calidad realizado en 1952 centros de salud urbanos y rurales, y 214 hospitales generales de la Secretaría de Salud. ${ }^{43}$ De hecho, en la Segunda Encuesta Nacional de Satisfacción con los Servicios de Salud, realizada por la Fundación Mexicana para la Salud en el año 2000, los entrevistados señalaron que los servicios de salud en su comunidad habían empeorado, en comparación con los que tenían en 1994 y los calificaron como "inferiores" a los servicios de las escuelas públicas. ${ }^{44}$

\section{Parámetro V. Financiamiento equitativo}

\section{Justicia en la contribución financiera}

Dentro de un sistema de salud equitativo es de esperarse que cada individuo contribuya al financiamiento de acuerdo con su capacidad de pago y que un episodio de mala salud no genere gastos excesivos.

En este rubro, a juzgar por los datos de un ejercicio de evaluación de 192 sistemas nacionales de salud recientemente publicados por la Organización Mundial de la Salud (OMS), el sistema mexicano presenta un desempeño pobre. En el componente de justicia financiera, que refleja tanto la incidencia de gastos catastróficos por motivos de salud como la progresividad del financiamiento del sistema de salud, México ocupó el lugar 144 a escala mundial, por arriba de Brasil y Chile, pero muy por debajo de Costa Rica y Colombia. ${ }^{21}$

Este mal desempeño se debe al hecho de que más de la mitad de la población mexicana carece de seguro de salud. Esta población se ve obligada frecuentemente a enfrentar sus necesidades de salud con gastos de bolsillo. Se calcula que en México alrededor de 50\% del gasto total en salud es gasto privado y casi la totalidad de este gasto es de bolsillo. ${ }^{45}$ En Colombia esta cifra asciende a $25 \%$ y en Gran Bretaña a 3\%. ${ }^{45}$

Puesto que siempre existe un elemento de incertidumbre en la pérdida de la salud, la falta de previsión representada por el alto pago de bolsillo deja a las familias mexicanas expuestas al riesgo de enfrentar gastos catastróficos por motivos de salud. De acuerdo con un análisis de la Encuesta Nacional de Ingreso-Gasto de los Hogares realizado en el año 2000, entre 2 y 3 millones de hogares mexicanos se ven obligados a utilizar más de la tercera parte de su ingreso disponible (gasto total menos gasto en alimentos) para hacer frente a sus necesidades de salud, incurriendo así en gastos potencialmente catastróficos que los pueden llevar a la pobreza o sumirlos todavía más en ella. ${ }^{22}$ Este fenómeno es particularmente frecuente entre las familias de menores ingresos que carecen de seguro médico. 
Los gastos de bolsillo, además, son regresivos. En México, los hogares con mayores recursos utilizan un menor porcentaje de su ingreso en la atención de sus necesidades de salud a través de gastos de bolsillo que los hogares pobres. ${ }^{22}$

La regresividad también es característica de los servicios de salud de la seguridad social. Según Scott, los subsidios públicos a la seguridad social se concentran principalmente en los hogares con mayores recursos. Los deciles de ingresos VIII, IX y X concentran 13.9, 15.3 y $11.4 \%$ de los subsidios al IMSS, respectivamente, mientras que los deciles I y II concentran 4.8 y $3.4 \%$, respectivamente. ${ }^{46}$

En este rubro la reforma de 1995-2000 se limitó a ofrecer el Seguro para la Familia, que al ampliar el acceso a la seguridad social, seguramente redujo entre las pocas familias beneficiadas el gasto de bolsillo $y$, por lo tanto, los gastos catastróficos por motivos de salud.

\section{Parámetro VI. Eficiencia distributiva, efectividad y calidad de la atención}

La equidad está estrechamente relacionada con un uso adecuado de los recursos. Los desperdicios afectan sobre todo a aquellas personas cuyas necesidades de salud están apenas satisfechas. Así, cualquier propuesta de reforma que busque la equidad deberá preocuparse por garantizar una mezcla adecuada de intervenciones y un énfasis en prácticas basadas en evidencia $\mathrm{y}$ en mecanismos que garanticen la efectividad de la atención.

\section{Enfasis en la atención de primer nivel}

El gasto público en México tradicionalmente ha favorecido la atención curativa. Entre 1992 y 1997, alrededor de $70 \%$ del gasto total en salud se dedicó a atención curativa y menos de $10 \%$ a actividades de salud pública. ${ }^{47}$ La proporción del gasto dedicada a la atención curativa de hecho se incrementó en los años de reforma. El gasto en este tipo de atención, además, se concentró en la atención de especialidad. En el IMSS, por ejemplo, los hospitales de segundo y tercer nivel de atención absorbieron cerca de $75 \%$ del presupuesto de esta institución. ${ }^{47}$

Además de esta concentración del gasto en la medicina de especialidad, las instituciones de salud han desarrollado pocos incentivos para mantener a los médicos en el primer nivel de atención. Dentro de los pocos incentivos destacan el fortalecimiento, dentro de los programas de enseñanza, de la especialidad en medicina familiar y atención comunitaria. La Se- cretaría de Salud y el ISSSTE adoptaron, además, en años recientes, una política salarial igualitaria entre niveles de atención como estímulo a los médicos familiares.

\section{Prácticas basadas en evidencia}

Las iniciativas de reforma desarrolladas en la década de 1990 no incorporaron la promoción de la práctica clínica o de la salud pública basada en evidencias. Aunque en algunos círculos clínicos se crearon grupos que promovieron la "medicina basada en evidencia", su influencia ha sido muy limitada.

\section{Certificación de los profesionales de la salud}

Desde 1943 las licencias profesionales de la salud han sido responsabilidad de las instituciones de educación superior y de la Dirección General de Profesiones de la Secretaría de Educación Pública. En 1994, además, se creó el Centro Nacional para la Evaluación de la Educación, que diseñó el Examen Nacional de Calidad Profesional para diversas profesiones. Este examen se está aplicando ya en varias escuelas de medicina como requisito de licenciamiento y en otras como opción de graduación.

La certificación de especialistas la controlan los consejos de las diversas especialidades médicas, que para esta tarea específica funcionan bajo la coordinación de las Academias Nacional de Medicina y Mexicana de Cirugía. Esta certificación está siendo utilizada por instituciones públicas y privadas, así como por las compañías de seguros, como requisito para la contratación de médicos especialistas.

En 1996 el Colegio Nacional de Enfermeras y la Federación Mexicana de Asociaciones de Facultades y Escuelas de Enfermería crearon el Sistema Nacional de Acreditación y Certificación de Enfermería. Este sistema de validación, sin embargo, todavía no entra plenamente en funciones.

Otra tarea pendiente en esta área es la regulación de los practicantes de medicinas alternativas, que, con excepción de los homeópatas y quiroprácticos, por lo general no pasan por procedimientos formales de capacitación y certificación.

\section{Medidas de acreditación de planes y hospitales}

Hasta mediados de los años noventa, la "certificación" de las unidades de salud se había limitado a la obtención de un registro sanitario. Sin embargo, en 1998 se echó a andar un proceso que culminó con la creación de la Comisión Nacional para la Certificación de 
Hospitales, que se hizo depender del Consejo de Salubridad General. Esta Comisión se encargó de supervisar la acreditación voluntaria de las unidades públicas y privadas de salud que llevan a cabo diversas agencias privadas haciendo uso de estándares consensuados de estructura, proceso y resultado. A finales del año 2000, alrededor de 500 hospitales habían solicitado su evaluación. ${ }^{22}$

\section{Participación comunitaria en la búsqueda} de la calidad de la atención

Posiblemente el único mecanismo a través del cual la "comunidad" ha participado en años recientes en el monitoreo de la calidad de la atención son las encuestas de satisfacción con los servicios de salud. El IMSS aplica en sus unidades este tipo de encuesta, cuyos resultados le sirven para hacer ajustes en sus procedimientos organizacionales y en los procedimientos de atención clínica. Asimismo, la Fundación Mexicana para la Salud ha llevado a cabo Encuestas Nacionales de Satisfacción con los Servicios de Salud, una en 1994 y otra en el año 2000. Estas encuestas han permitido tener una idea general de la percepción que tienen los usuarios tanto de los servicios públicos como privados.

\section{Parámetro VII. Valor por el dinero- eficiencia financiera}

\section{Minimización de gastos de administración}

Aunque existe una creciente conciencia sobre la necesidad de dirigir la mayor proporción de recursos a actividades que mejoren de manera evidente las condiciones de salud de la población, la reforma del sistema mexicano de salud ha hecho poco por afrontar de manera explícita el problema de los gastos administrativos excesivos. Las cifras de 1995 indican que la Secretaría de Salud y el ISSSTE dedicaban 25 y 33\%, respectivamente, de sus recursos a actividades administrativas. ${ }^{48}$ Ninguna de las iniciativas desarrolladas en el sector entre 1994 y 2000 se dirigieron a combatir este tipo de ineficiencias.

\section{Compras que reduzcan costos}

Las compras consolidadas de medicamentos y otros insumos se incorporaron a las instituciones mexicanas de salud desde principios de los años ochenta. La centralización de estas compras trajo consigo ahorros importantes, pero también problemas de abasto que a la fecha no se han podido resolver. La consumación de la descentralización de los servicios de salud para población no asegurada en la segunda mitad de los años noventa le permitió a los estados abstenerse de participar en las compras consolidadas nacionales si así convenía a sus intereses.* Esto ha permitido el surgimiento en algunas entidades de nuevos mecanismos de compra consolidada estatal o regional que posiblemente han generado ahorros importantes, al tiempo que se acerca al proveedor de insumos a las unidades de atención.

\section{Minimización del desplazamiento de costos}

Uno de los problemas más serios de la segmentación del sistema mexicano de salud es el desplazamiento de costos. Este problema no se ha podido resolver. Las cifras de las Encuestas Nacionales de Salud 1994 y 2000 indican que la frecuencia de uso de servicios de la Secretaría de Salud por la población derechohabiente no se ha modificado. ${ }^{49}$ El uso de servicios de la Secretaría de Salud de un estado por habitantes de otra entidad federativa es también un fenómeno muy común. El ejemplo más ilustrativo es el de los residentes del Estado de México, que de manera regular hacen uso de los servicios de salud para población no asegurada del Distrito Federal.

Las iniciativas de reforma que se dieron en los años noventa no incluyeron el diseño de mecanismos de pago cruzado entre instituciones y unidades que limitaran el desplazamiento de costos e hicieran más transparente el traslape de la demanda.

\section{Medidas antifraude y antiabuso}

A pesar de los frecuentes abusos que, se sabe, se dieron sobre todo en el área de compra de medicamentos e insumos, en la década de los noventa prácticamente no se tomaron medidas explícitas de combate a la corrupción en el sector salud.

\footnotetext{
* La descentralización de los servicios de salud para población no asegurada se inició a principios de la década de los ochenta, se interrumpió a finales de esa misma década y se concluyó a finales de los años noventa. Este accidentado proceso por fortuna culminó con la total descentralización de los recursos y responsabilidades de prestación de servicios a las entidades federativas. En el año 2000, estas entidades federativas podían ya decidir localmente sobre la distribución de todos los recursos del llamado Ramo 33 (Fondo de Aportaciones a los Servicios de Salud o FASSA), que en ese año representaron casi $60 \%$ de los recursos federales dedicados a atender las necesidades de salud de la población no asegurada.
} 


\section{Parámetro VIII. Responsabilidad ante el público}

Procedimientos explícitos, públicos y detallados para la evaluación de los servicios, con informes públicos

La evaluación del desempeño de los servicios de salud fue una preocupación constante de las instituciones del sector salud en los años noventa. En 1995 se crea dentro de la Secretaría de Salud la Dirección General de Evaluación y Seguimiento. Sin embargo, los productos de las iniciativas de evaluación no se hicieron públicos ni circularon entre los responsables de los servicios y programas que pudieron haberse beneficiado con ellos. Este es el caso, por ejemplo, del PASSPA y del PAC, que aunque contaron con evaluaciones exhaustivas, sus resultados nunca se dieron a conocer. ${ }^{50}$

Procedimientos explícitos y democráticos para la asignación de recursos

Uno de los principales problemas del sistema mexicano de salud es la enorme desigualdad que existe en la distribución de recursos entre agencias, entidades federativas y grupos de ingreso. Los recursos en general han tendido a favorecer a las instituciones que cubren a las poblaciones de importancia estratégica y a las entidades con mayores capacidades de negociación.

En un intento por revertir este proceso, a finales de los años noventa se diseñó una fórmula destinada a promover la equidad en la asignación de recursos en la Secretaría de Salud. El principal avance de esta fórmula es que vinculó de manera explícita la asignación presupuestal con las necesidades de salud. Sin embargo, esta fórmula se utilizó para distribuir únicamente los recursos adicionales que pudieran movilizarse en esta Secretaría.

Procedimientos justos para quejas y reclamos

Hasta bien entrada la década de los noventa, los usuarios de los servicios de salud contaban con pocas instancias para la presentación de quejas o querellas: los departamentos de quejas de las instituciones públicas, las comisiones de derechos humanos y, muy ocasionalmente, los tribunales. En 1996, sin embargo, se creó la Comisión Nacional de Arbitraje Médico (Conamed), cuyo objetivo es contribuir a la solución de controversias entre usuarios y proveedores públicos y privados de servicios de salud a través de procedimientos más simples que las demandas legales. Hasta finales del año 2000, la Conamed había atendido más de 15 mil asuntos y al parecer su desempeño había sido aceptable..$^{51}$

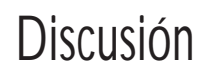

Las medidas de reforma del sistema mexicano de salud que se llevaron a cabo en la segunda mitad de la década pasada tenían como objetivos mejorar la calidad, eficiencia y equidad de los servicios públicos de salud. Destacan dentro de estas iniciativas las siguientes:

- La introducción de un nuevo esquema financiero dentro del IMSS que implicó un incremento muy importante de la participación gubernamental en su presupuesto.

- La desconcentración del IMSS mediante la creación de 139 áreas médicas que están recibiendo, desde enero de 1998, asignaciones presupuestales sobre la base de una fórmula capitada ajustada por edad y sexo.

- La promoción de la afiliación al IMSS de los miembros de la economía informal con capacidad de pago, a través de un nuevo plan (Seguro para la Familia) financiado con contribuciones de los trabajadores y un subsidio federal.

- La conclusión del proceso de descentralización de los servicios de salud para la población no asegurada iniciado en 1986.

- La entrega de un paquete de 12 intervenciones, a través del PAC, a los 10 millones de mexicanos que hasta 1995 no contaban con acceso regular a servicios básicos de salud.

En general, estas medidas tuvieron un impacto positivo en el acceso de los pobres a la atención de su salud. La oferta de un seguro de salud para los autoempleados y los miembros de la economía informal con capacidad de pago amplió el acceso de un número pequeño, pero no despreciable de hogares a servicios integrales de una calidad aceptable, y en la medida en la que amplió el prepago, redujo el riesgo de gastos catastróficos por motivos de salud. La principal limitación de esta iniciativa es la imposibilidad de extenderla a un número considerable de hogares debido al monto de la contribución familiar, que es inalcanzable para los más de 35 millones de mexicanos que viven en condiciones de pobreza o pobreza extrema.

El PAC redujo considerablemente las barreras financieras y diversas barreras no financieras (geográficas, de lengua, culturales) a las que se enfrentaban las poblaciones más pobres del país y contribuyó a reducir la mala distribución de algunos recursos para la 
salud. Su reto ahora es ampliar el paquete de beneficios, y mejorar la calidad y la oportunidad de la atención. Las 12 intervenciones permitieron atender problemas que son casi exclusivos de los pobres. Sin embargo, estas intervenciones son insuficientes para una población que sufre ya también de problemas crónicos y degenerativos que requieren de atención más especializada. Por el lado de la calidad, es importante señalar que se ha demostrado la existencia de un problema serio y crónico de abasto de medicamentos en las unidades de este programa..$^{52}$ Asimismo, la oferta de servicios a través de brigadas móviles, aunque ha contribuido a ampliar la cobertura, tendrá que irse sustituyendo por una oferta más regular.

La descentralización de los servicios de salud para población no asegurada y la desconcentración del IMSS han permitido acercar la toma de decisiones a los sitios de operación de los servicios y, en esa medida, han contribuido a fortalecer la imparcialidad del sistema. Estos procesos también parece que han incrementado la eficiencia financiera del sistema al facilitar el manejo de los recursos y la compra de insumos. El reto en estos casos es poder extender paulatinamente la toma de decisiones local al interior de las entidades federativas y al interior de las delegaciones del IMSS.

Dentro de las medidas tomadas en la segunda mitad de la década de los noventa que influyeron también de manera positiva en la equidad e imparcialidad del sistema de salud se incluyen las siguientes (cuadro V):

- Algunas actividades intersectoriales dirigidas a mejorar la vivienda y la nutrición básica de las poblaciones más pobres (Progresa);

- Diversas medidas dirigidas a incrementar la efectividad y calidad de la atención, como la certificación de los profesionales de la salud, la certificación de las unidades de salud y el uso incipiente, pero cada vez mayor, de encuestas de satisfacción de los usuarios con los servicios de salud;

- El uso de una fórmula que hace explícitos los criterios de asignación de recursos en la Secretaría de Salud, y

- El establecimiento de la Conamed, que sin duda hará que los prestadores de servicios de salud sean más cuidadosos y los usuarios más concientes de sus derechos.

Por el lado de los pendientes, las iniciativas de reforma poco pudieron hacer por la justicia del financiamiento, la mala distribución de recursos, las barreras de género, la segmentación de la atención, la concentración en la atención curativa de segundo y tercer ni- vel, y la rendición de cuentas y la transparencia. En el ámbito intersectorial poco se hizo por atender el creciente problema de la violencia y la seguridad pública, y prácticamente no se hizo nada por documentar las inequidades en salud.

El gasto de bolsillo sigue siendo muy alto en México y el porcentaje de su ingreso que por esta vía dedican los hogares más pobres a atender sus necesidades de salud es mayor que el porcentaje que dedican las familias con mayores recursos. Además, el subsidio que reciben las familias de mayores ingresos de parte de las instituciones de seguridad social es considerablemente mayor que el subsidio que reciben las familias pobres. No debe sorprender, por lo tanto, el lugar que ocupó México en el rubro de protección financiera en las evaluaciones de la OMS.

Por lo que se refiere a las brechas en recursos, aunque el PAC contribuyó en cierta medida a reducirlas, éstas siguen siendo enormes, tanto entre instituciones como entre entidades federativas y grupos sociales. Las diferencias en el gasto per cápita entre instituciones, en el número de médicos por 1000 habitantes entre entidades federativas o en acceso a la atención del parto entre municipios con diferentes niveles de marginación, por ejemplo, poco se modificaron en los últimos años de la década pasada.

En cuanto a la minimización de barreras de género, existen serios rezagos como lo indica el alto número de defunciones maternas por causas obstétricas directas y los pobres resultados del Programa de Detección Masiva del Cáncer Cérvico Uterino.

Un problema persistente del sistema de salud es su segmentación. Las instituciones de seguridad social son las que tienen un mayor gasto per cápita. Esta desigualdad por supuesto que se refleja en la calidad de la atención, que es más deficiente en los servicios para la población no asegurada.

La reforma tampoco enfrentó la creciente concentración de los recursos en la atención curativa de segundo y tercer nivel. De hecho, fuera de la preocupación por las enfermedades prevenibles por vacunación, son muy pocos los servicios preventivos que ofrece el sistema mexicano de salud en su conjunto. Es de llamar la atención, por ejemplo, lo poco que se hizo en el terreno de la promoción de la salud, a pesar de la creciente importancia de las enfermedades no transmisibles, en las que los hábitos de vida juegan un papel fundamental.

Por último, es muy importante destacar la ausencia casi total de mecanismos de rendición de cuentas y transparencia. Entre 1995 y 2000 poco se hizo en el terreno de la evaluación, al grado de que en 1998 desaparece del organigrama de la Secretaría de Salud la Dirección 


\section{Cuadro V \\ Calificaciones otorgadas a los ParÁmetros para la eVAluación de La EQUidad E IMPARCIALIDAD DE LA REFORMA DEL SISTEMA MEXICANO DE SALUD}

Parámetro

Calificación

Parámetro I. Salud pública intersectorial

- Nutrición básica

- Vivienda

- Factores ambientales

- Educación y educación para la salud

- Violencia y seguridad pública

- Información para monitorear la inequidad en salud

Parámetro II. U niversalidad en el acceso, minimización de barreras no financieras

- Cobertura del sector informal

- Aseguramiento del sector formal

\begin{tabular}{cc}
\multicolumn{2}{c}{ Calificación } \\
\hline & $+1++$ \\
\hline++ & \\
++ & \\
++ & \\
+ & \\
- &
\end{tabular}

Parámetro III. Universalidad en el acceso, minimización de barreras no financieras

- Minimización de la mala distribución de recursos t

- Minimización de las barreras de género

- Minimización de las barreras de lengua, culturales y de clase

$++$

Parámetro IV. Beneficios integrales y uniformes

- Integralidad: acceso a todos los servicios necesarios y efectivos que se consideran financiables

- Reducción del escalonamiento y calidad uniforme

Parámetro V. Financiamiento equitativo

- Justicia en la contribución financiera

$\frac{d+}{++}$

Parámetro VI. Eficiencia distributiva, efectividad y calidad de la atención

- Enfasis en la atención de primer nivel -

- Prácticas basadas en evidencia

- Certificación de los profesionales de la salud $\quad++$

- Medidas de acreditación de planes y hospitales

- Participación comunitaria en la búsqueda de la calidad de la atención

Parámetro VII.Valor por el dinero-eficiencia financiera

$+$

- Minimización de gastos de administración

- Compras que reduzcan costos t+

- Minimización del desplazamiento de costos -

- Medidas antifraude y antiabuso

Parámetro VIII. Responsabilidad ante el público

- Procedimientos explícitos, públicos y detallados para la evaluación de los servicios

$+$

- Procedimientos explícitos y democráticos para la asignación de recursos

- Procedimientos justos para quejas y reclamos

General de Evaluación y Seguimiento. Además, los resultados de los ejercicios de evaluación que se llevaron a cabo en la última década nunca se hicieron públicos ni se pusieron a disposición de los tomadores de decisiones que pudieron haberse beneficiado de ellos.

Para concluir, podemos afirmar que la reforma del sistema mexicano de salud de la segunda mitad de los años noventa tuvo cierto impacto sobre algunos aspectos de la equidad -sobre todo los relacionados con el acceso a servicios esenciales de salud- y la eficiencia administrativa -en particular lo relacionado con el proceso de descentralización-, pero un efecto limitado sobre la justicia del financiamiento y el gobierno democrático. 


\section{A gradecimientos}

Quisiéramos agradecer los comentarios de Norman Daniels y Walter Flores a la adaptación de los parámetros a la realidad mexicana. También quisiéramos hacer explícito nuestro agradecimiento a las personas que nos ayudaron a definir los indicadores para cada parámetro: Bernardo Hernández, Mauricio Hernández, Martha Híjar y Ana Langer. La responsabilidad por el contenido de este artículo es exclusiva de los autores.

\section{Referencias}

1. De la Torre R. Pobreza. N exos 2002;24(289):20-21.

2. Székely M. D esigualdad. N exos 2002;24(289):18-20.

3. Poder Ejecutivo Federal. Programa de Reforma del Sector Salud 1995-2000. México, DF: Poder Ejecutivo Federal; 1995.

4. Daniels $N$, Light DW, C aplan RL. Benchmarks of fairness for health care reform. N ew York: 0 xford U niversity Press; 1996.

5. Knowles JC, Leigthen C, Stinson W. Indicadores de medición del desempeño del sistema de salud.W ashington, DC: Iniciativa Regional del Sector Salud en América Latina y El Caribe; 1997.

6. Jee $M, O r Z$. Health outcome measurement in OECD countries:

Toward outcome-oriented policy making. París: 0 rganization for Economic Cooperation and Development; 1999.

7. Murray $C$, Frenk J.A framework for assessing the performance of health systems. Bull W orld Health 0 rgan 2000;78(6):717-731.

8. Hsiao W C, Roberts MJ, Berman PA, Reich MR. Encauzando la reforma en el sector salud. Santiago de Chile: Bitrán y Asociados, Instituto del Banco Mundial; 2001.

9.W hitehead M.The concepts and principles of equity and health. Copenhague:W HO Regional 0 ffice; 1990.

10. Dahlgren $G, W$ hitehad M. Policies and strategies to promote equity in health. Copenhague:W HO Regional 0 ffice; 1991.

11. Rawls J. Justicia como equidad. Madrid: Editorial Tecnos; 1999. 12. D aniels N, Bryant J, Castano RA, Gómez-D antés 0 , Khanz KS, Pannarunother $S$. Benchmarks of fairness for health care reform: $A$ policy tool for developing countries. Bull W orld Health 0 rgan 2000:78(6):740-750.

13. Gertler P, Boyce S.A experiment in incentive-based welfare:The impact of PRO GRESA in Mexico (working paper). W ashington, DC: W orld Bank; 2000.

14. Rivera-D ommarco J, Shamah-Levy T,Villalpando-Hernández S, González de Cossío T, Hernández-Prado B, Sepúlveda J, ed. Encuesta $\mathrm{N}$ acional de Nutrición 1999. Cuernavaca, Morelos, México: Instituto $N$ acional de Salud Pública; 2001.

15. Instituto N acional de Estadística, G eografía e Informática. X II Censo General de Población y Vivienda 2000. Tabulados de la muestra censal. México, DF: IN EG I; 2002.

16. Secretaría de Salud. Encuesta N acional de Adicciones. México, D F: SSA; 1998

17. Secretaría de Salud. Mortalidad. México, DF: SSA; 1990 y 1999. 18. Prescott-A llen R.The wellbeing of nations. A country-by-country index of quality of life and the environment. W ashington, DC: Island Press, ID RC; 2001

19. Secretaría de Salud. A nuario Estadístico. México, DF: SSA; 1999. 20. Secretaría de Salud. Salud: México 2003. México, DF:SSA; 2004. 21. World Health 0 rganization. W orld Health Report 2000. Health systems: Improving performance. G inebra:W HO ; 2000.
22. Secretaría de Salud. Programa N acional de Salud 2001-2006. La democratización de la salud en México. México, DF: Secretaría de Salud; 2001.

23. Consejo Nacional de Población. Indices de marginación 2000. México, DF: Conapo; 2001.

24. Comisión $\mathrm{N}$ acional del Agua. Compendio básico del agua en México. México, DF; 2002.

25. Instituto Nacional de Ecología, Secretaría del Medio A mbiente y Recursos N aturales. Programa de Mejoramiento de la Calidad del Aire en el Valle de México 1995-2000. México, D F: Semarnap; 1995.

26. Gutiérrez V. Presentación en el Segundo Encuentro Latinoamericano sobre Calidad del Aire y Salud; 2000 octubre 14; México, DF, México. Disponible en: http://www.cepis.ops-oms.org/bvscie/e/fulltext/2encuent/ mexico1.pdf. [2004 marzo 23].

27. Comisión Económica para A mérica Latina. Seguridad ciudadana y violencia. Santiago de Chile: CEPAL; 1999.

28. Sistema N acional de Salud. Boletín de información estadística 2000. México, DF: Dirección General de Información y Evaluación del Desempeño, Secretaría de Salud; 2000 y 2001.

29. Secretaría de Educación Pública. Plan y Programas de Estudios de Educación Secundaria. México, DF: SEP; 2002. D isponible en: www.sep.gob.mx. [2003 nov 20].

30. C O N A SIDA, Secretaría de Salud. Programas de Prevención. 2002.

Disponible en: www.ssa.gob.mx/conasida/index.htm. [2003 noviembre 20]. 31. Secretaría de Salud, Consejo N acional contra las Adicciones. Lineamientos para el reforzamiento del Programa $\mathrm{N}$ acional contra las Adicciones. 2000. Disponible en: www.ssa.gob.mx/unidades/conadic. [2003 noviembre 20].

32. Secretaría de Salud. Programa de Ampliación de Cobertura. Lineamientos de operación. México, DF: SSA; 1996.

33. Gómez-Dantés 0 . Health reform and policies for the poor in Mexico. En: Lloyd-Sherlock P, ed. Healthcare reform and poverty in Latin America. Londres: University of London; 2000:128-142.

34. Instituto Mexicano del Seguro Social. Memoria estadística. México, DF: IMSS, D irección de Planeación y Finanzas; 2000.

35. Sistema N acional de Salud. Boletín de información estadística 1995. México, DF: Dirección General de Estadística e Informática, Secretaría de Salud; 1996.

36. Gómez-D antés 0, Garrido-Latorre F, López-Moreno S,Villa B, López-Cervantes M. Evaluación de un programa de salud para población no asegurada. Rev Saude Publica 1999;33(4):401-412.

37. Sistema $\mathrm{N}$ acional de Salud. Boletín de Información Estadística 1999. México, DF: Dirección General de Información y Evaluación del D esempeño, Secretaría de Salud; 2001.

38. Secretaría de Salud. Salud: México 2003. Información para la rendición de cuentas. México, DF: Dirección General de Información y Evaluación del Desempeño, Secretaría de Salud; 2004.

39. Lazcano E, N ájera P,Alonso P, Buiatti E, Hernández M. Programa de Detección 0 portuna de $C$ áncer $C$ érvico-Uterino en México. Diagnóstico situacional. Rev Inst N ac C ancerol 1996;24(3):123-140. 40. Valdés LM. Los indios en el tercer milenio. Ciencias 2001;(6061):128-132.

41. Sánchez-Pérez HJ, 0 choa-Díaz H, N avarro A, Martín-Mateo M. La atención del parto en Chiapas, México: ¿dónde y quién los atiende? Salud Publica Mex 1998:40:494-502.

42. Comisión $\mathrm{N}$ acional de Arbitraje Médico. Cuarto Informe de Actividades: Junio 1999-Mayo 2000. México, DF: Conamed; 2000. 43. Bobadilla JL. Q uality of perinatal medical care in Mexico City. México, DF: Instituto N acional de Salud Pública; 1988.

44. Fundación Mexicana para la Salud. Segunda Encuesta N acional de Satisfacción con los Servicios de Salud. México, DF: Funsalud; 2000. 45. Hernández P, Zurita B, Ramírez R, Alvarez F, Cruz C. Las cuentas nacionales de salud. En: Frenk J, ed. 0 bservatorio de la Salud. 
Necesidades, servicios políticas. Mexico, D F: Fundación Mexicana para la Salud; 1997:119-142.

46. Scott J. La otra cara de la reforma fiscal: la equidad del gasto público. México, DF:CIDE; 2001.

47. Secretaría de Salud. Sistema de Cuentas $N$ acionales y Estatales de Salud. México, DF: Dirección General de Información en Salud, Secretaría de Salud; 2002.

48. Frenk J, González-Block MA, Lozano R y col. Economía y salud. México, DF: Fundación Mexicana para la Salud; 1994.

49. Instituto $\mathrm{N}$ acional de Salud Pública. Encuesta N acional de Salud 2000. Cuernavaca, Morelos, México: IN SP; 2002.
50. Gómez-D antés 0 . ¿Q ué hacemos con la salud de los pobres? Este País 2001;(122):26-31.

51. Comisión N acional de Arbitraje Médico. Cuarto Informe de Actividades. México, DF: Conamed; 2000.

52. Gómez-D antés O, Garrido-Latorre F,T irado-Gómez L, Ramírez D. A bastecimiento de medicamentos en unidades de primer nivel de atención de la Secretaría de Salud de México. Salud Publica Mex 2001;43(3):224-232. 